\title{
Resolving the microcosmos of complex samples: UPLC/travelling wave ion mobility separation high resolution mass spectrometry for the analysis of in vivo drug metabolism studies
}

\author{
Stefan Blech • Ralf Laux
}

Received: 1 August 2012 /Revised: 24 October 2012 / Accepted: 5 November 2012 / Published online: 29 November 2012

(C) The Author(s) 2012. This article is published with open access at Springerlink.com

\begin{abstract}
In vivo drug metabolism studies with low concentrations of analytes and high matrix burden are challenging. Of special interest are 'first-in-man' studies in early stages of pharmaceutical development that do not use ${ }^{14} \mathrm{C}$ labeled drug candidates. Beside conventional MS-fishing techniques which are biased towards known/expected metabolites and mass defect filtration procedures, this paper focuses on the untargeted/unbiased analysis of drug related compounds in complex matrices using two orthogonal separation techniques: UPLC and TWIMS. Standard sample material after oral administration of a drug compound to rats was investigated by UPLC/ TWIMS in $\mathrm{MS}^{\mathrm{E}}$ acquisition mode using interlaced collision energies for the parallel detection of $[\mathrm{M}+\mathrm{H}]^{+}$parent ions and fragments. Due to the fragmentation after ion mobility separation in the transfer region of the Synapt G2-triwave device, $[\mathrm{M}+\mathrm{H}]^{+}$ion species are aligned with their related fragments by virtue of possessing the same retention time and drift time profile. Four dimensional data analysis of the continuum raw data was performed by automated peak picking and alignment within the $\mathrm{MS}^{\mathrm{E}}$ viewer software. As result, completely purified MS- and MS/MS-data of metabolites were extracted from raw mass data with high matrix burden and were used without compromise for structure elucidation. This analytical methodology is universally applicable for the unbiased/untargeted and robust analysis of any analyte of interest in complex matrices, including small molecules, peptides and proteins. The high quality data files can be used as data repositories for the purpose of retrospective analysis which is of particular interest for the long term process in drug development.
\end{abstract}

\footnotetext{
S. Blech $(\bowtie) \cdot$ R. Laux

Department of Drug Metabolism and Pharmacokinetics,

Boehringer Ingelheim Pharma GmbH \& Co KG,

88397 Biberach, Germany

e-mail: stefan.blech@boehringer-ingelheim.com
}

Keywords Drug metabolism - Complex sample material . Ion mobility separation · 4D-data analysis · Data repository · MSE-analysis

\section{Introduction}

Drug metabolism studies are challenging. This is especially true for the screening for drug related compounds in complex samples of in vivo studies with high matrix burden including the routinely used samples of plasma, urine, bile, and faeces, but also of whole blood and of extracts of organs.

Depending on applied drug doses and the pharmacokinetics of the analytes, signals of interest with exceptionally low intensity need to be screened out of matrix interferences of endogenous and/or co-administered additives with high signal intensity. Additionally the absence of radiotracers, which is the case in 'first-in-man' studies, results in challenging situations when metabolites needed to be reliably identified and elucidated in early stages of pharmaceutical development. With the background of regulatory guidelines $[1,2]$ and the continuous progress of analytical technologies the pivotal role of $14 \mathrm{C}$-metabolism studies was recently revisited with the proposal that regulatory requirements can be even better met without conducting the traditional animal radiolabelled studies [3]. However the discussion evolves, it underlines the increasing importance of reliable high sensitivity screening methodologies in 'real life samples', especially in cold in vivo samples, for the future drug development process.

Many analytical approaches using different mass spectrometry techniques have been developed for the screening and identification of drug related compounds in drug metabolism studies. Unit resolution platforms like triple 
<smiles>C=CN(C)C(CCO)CCO</smiles>

Fig. 1 Metabolism pathways of M0

quadrupole [4] or ion trap instruments [5-8] used targeted approaches which required multiple runs of samples or predicted MRM- or untargeted enhanced survey scans to trigger product ion scanning within one analytical run. The data were successfully used for both metabolite identification and quantification of the parent drug and semi quantification of metabolites of in vivo and in vitro studies.

The high resolution hybrid systems, such as the LTQOrbitrap have effectively demonstrated their capabilities for parallel data collection, mass defect filtration and exact mass ion extraction for both in vitro and in vivo drug metabolism studies with and without data/information dependent acquisition methodologies [9-22]. With sophisticated generic settings including peak intensity dependent triggering and inclusion/exclusion list dependent triggering of MS/MS or

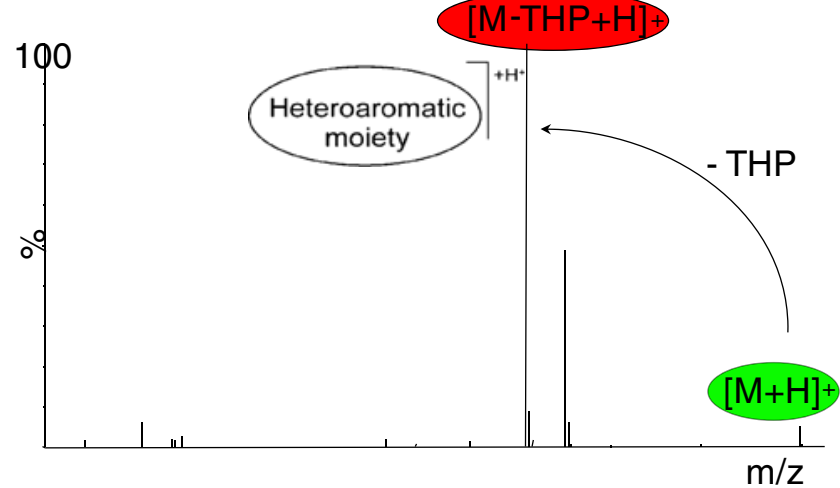

Fig. 3 MS/MS data of the parent compound's $[\mathrm{M}+\mathrm{H}]^{+}$

$\mathrm{MS}^{\mathrm{n}}$ experiments, in vivo drug metabolism studies were performed with high reliability with respect to metabolite coverage.

The $\mathrm{MS}^{\mathrm{E}}$ acquisition technique represents another approach and provides a completely untargeted and unbiased methodology which can be used with fast acquisition hybrid quadrupole time-of-flight (QTOF) instruments in combination with the separation power of ultra high performance liquid chromatography (UPLC). The advantage of this technique is the simplified data collection which is (1) independent of exclusion lists for high abundant matrix interferences that may impair the efficiency of data dependent MS/MS-triggering and which is (2) independent of generic or predictive inclusion lists which are biased and therefore potentially not comprehensive. $\mathrm{MS}^{\mathrm{E}}$ data enable the analysis for any compound of interest, irrespective of its signal intensities or those of co-eluting matrix ions.

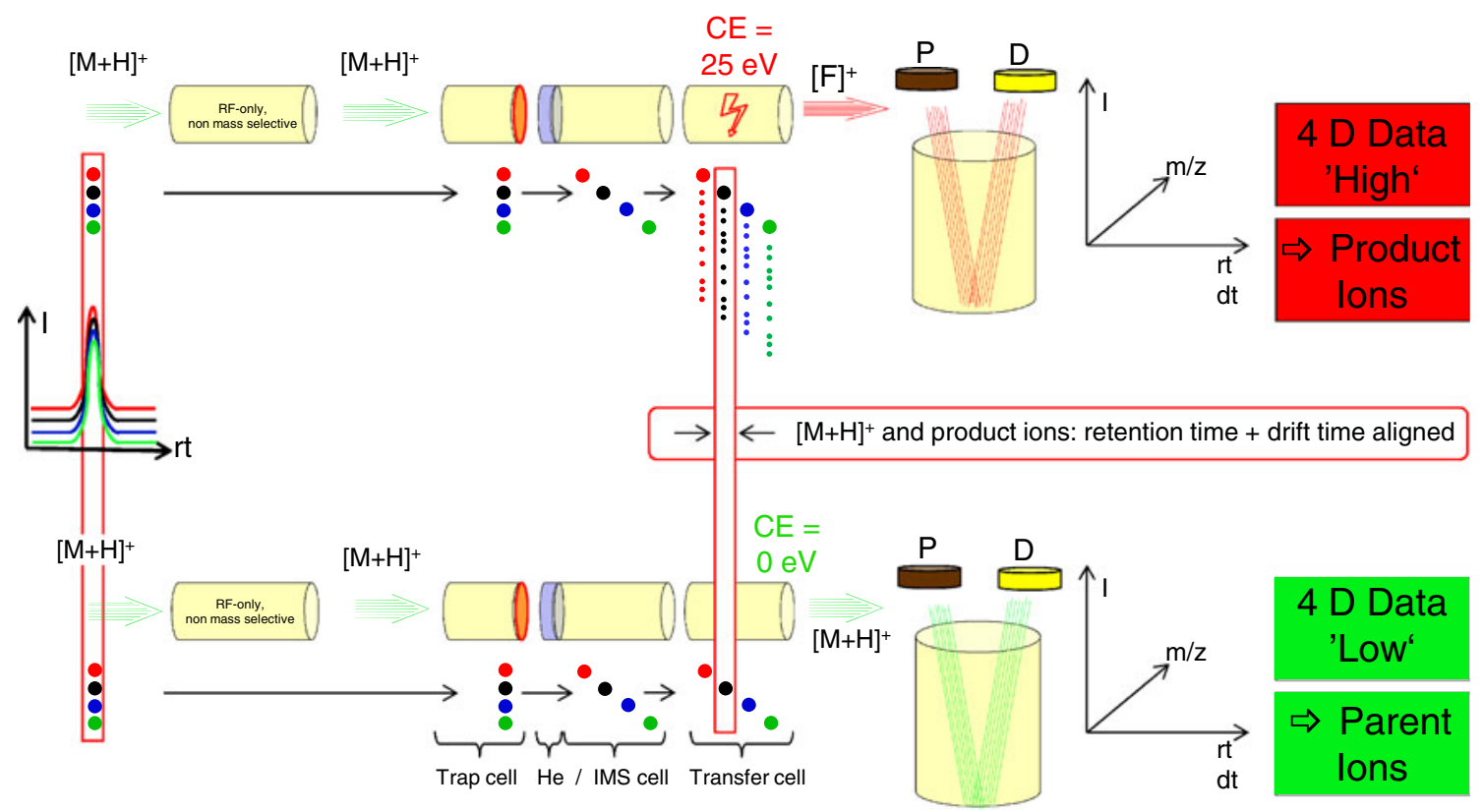

Fig. 2 Synapt G2, combined UPLC with IMS and MSE with interlaced collision energies 
This technology was previously shown to be universally applicable not only for drug metabolism studies [23-26], but also for impurity analysis [27], environmental/waste water analysis [28, 29], and proteomics [30]. We here report the use of the UPLC-QTOF/MS ${ }^{\mathrm{E}}$ approach as general 'first-line' screening for in vitro and in vivo studies. Published drug metabolism data of the recently approved drugs Dabigatran [31] and Linagliptin [32] were based on this technology. For both drugs the alignment of parent-ions with fragment ion species were achieved by matching the extracted ion chromatograms (XICs) with exactly the same peak shape. The chromatographic resolution therefore is an important factor in the process. The $\mathrm{MS}^{\mathrm{E}}$ approach perfectly works for samples with clean matrix from in vitro studies and with in vivo samples with advantageous analyte/matrix ratio. However, for the identification of low level metabolites dedicated MS/MS-experiments are needed due to strong interferences of co eluting matrix ions.

The utilization of ion mobility separation (IMS), as an additional principle of differentiation, will play a key role for the further development of the $\mathrm{MS}^{\mathrm{E}}$ acquisition methodology. Based on the collisional cross section as key parameter, ions
Fig. 4 Identification of drug related compound in rat urine by correlation of XICs of the diagnostic fragment $[\mathrm{M}-\mathrm{THP}+\mathrm{H}]^{+}$with metabolite $[\mathrm{M}+\mathrm{H}]^{+}$-ions. Upper trace: data 'high': XIC of

$[\mathrm{M}-\mathrm{THP}+\mathrm{H}]^{+}$, light gray:

TIC. Lower traces: data 'low': XIC of parent drug and metabolite's $[\mathrm{M}+\mathrm{H}]^{+}$, light gray: TIC
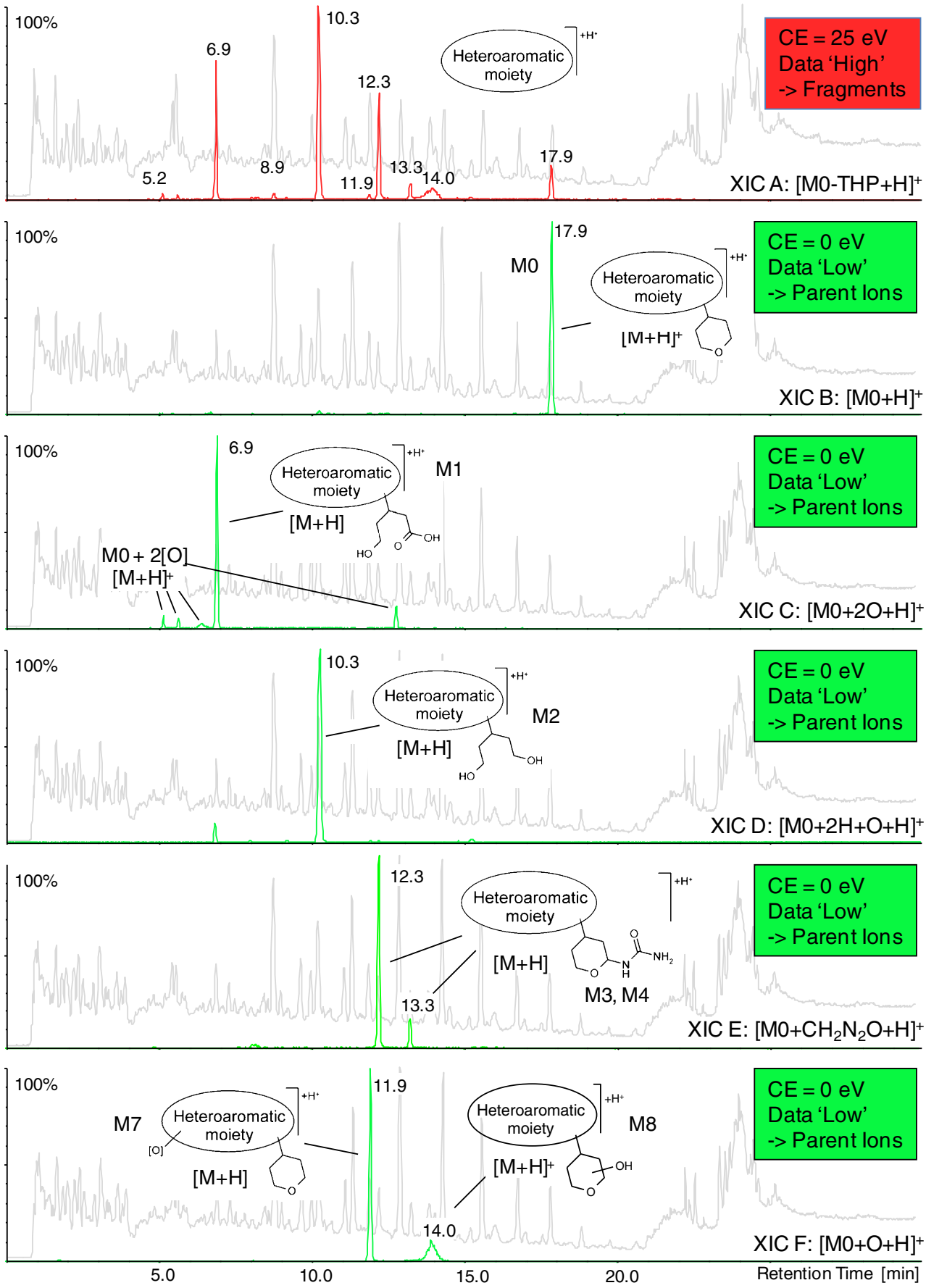
are separated by differences of individual size to charge ratios in combination with the interaction of a buffer gas. The ion mobility separation of structural isomers, polymeric conformers and enantiomers in applications of proteomics, glycomics and metabolomics was successfully demonstrated with various instrumental setups [33-35].

In this publication we present the combination of UPLC and travelling wave ion mobility separation (TWIMS) together with the $\mathrm{MS}^{\mathrm{E}}$ acquisition methodology as new approach for the reliable, unbiased and fast analysis of drugs and its metabolites which is tailored for the analysis in complex biological sample material.

Only parts of the parent compound structure and only reduced mass spectrometry data are allowed to be presented. However, this does not detract from the demonstration of the viability and utility of the general approach outlined here.

\section{Materials and methods}

Chemicals and reagents

The drug compound of interest (M0) was synthesized by the medicinal chemistry group of Boehringer Ingelheim Pharma GmbH \& Co. KG, Ingelheim, Germany. Chemical purity was $>97 \%$. For lock mass correction a solution of leucin enkephalin (Sigma Aldrich, Stadt, Germany) at a concentration of $2 \mu \mathrm{g} / \mathrm{ml}$ in acetonitrile $/ 0.1 \%$ aqueous formic acid (1:1) was used. Acetonitrile, methanol, water and formic acid were of analytical grade or higher purity and were obtained from Sigma Aldrich (Steinheim, Germany).
Biological sample material

The study protocol of in vivo experiments was in full conformance with the respective animal experimentation application approved by the local animal welfare officer of Boehringer Ingelheim Pharma $\mathrm{GmbH} \& \mathrm{Co}$. KG, Biberach, Germany, as well as by the responsible supervisory authority, Tübingen, Germany. Boehringer Ingelheim Pharma $\mathrm{GmbH} \& \mathrm{Co}$. KG is accredited by the Association for Assessment and Accreditation of Laboratory Animal Care International (AAALAC).

Male and female rats $(n=3$, stain: $\mathrm{Crl}: \mathrm{WI}(\mathrm{HAN}))$ received a single oral dose of $50 \mathrm{mg} / \mathrm{kg}$ of the drug compound dissolved in water after an overnight fast $(\geq 10 \mathrm{~h})$. Plasma (sampling times: $0,0.25,0.5,1,6,24 \mathrm{~h}$ ), urine and faeces (fractions 0-48 h) were sampled. Bile fractions (0-6 h) were obtained from bile duct cannulated rats $(n=2)$. Sample aliquots of urine and faeces samples of individuals were pooled proportionally according to the relative weight of individual sample fractions. Plasma was pooled proportionally to sampling times to yield a sample in which concentrations of analytes were proportional to AUC's (AUC = area under the concentration time curve) within the given time interval $[36,37]$. The pooled plasma $(0-24 \mathrm{~h})$ was processed by solid phase extraction using OASIS HLB cartridges (200 mg, $6 \mathrm{cc}$, Waters, Milford, MA, USA) and $0.1 \%$ aqueous formic acid/ acetonitrile (1:9) as eluent. Pooled faeces samples were exhaustively extracted with acetonitrile/water (1:1). Pooled urine and bile was diluted 1:10 with aqueous formic acid $(0.1 \%)$ before analysis. All samples and sample extracts were stored at $-20{ }^{\circ} \mathrm{C}$ until analysis.
Fig. 5 Urine after administration of M0. Upper trace: data 'high': XIC of $[\mathrm{M}-\mathrm{THP}+\mathrm{H}]^{+}$, light gray: TIC. Lower traces: data 'low': XIC of parent drug's and PEG's $[\mathrm{M}+\mathrm{H}]^{+}$, light gray: TIC, Inserted figure: mass data at $\mathrm{rt}=17.9 \mathrm{~min}$, data 'low'
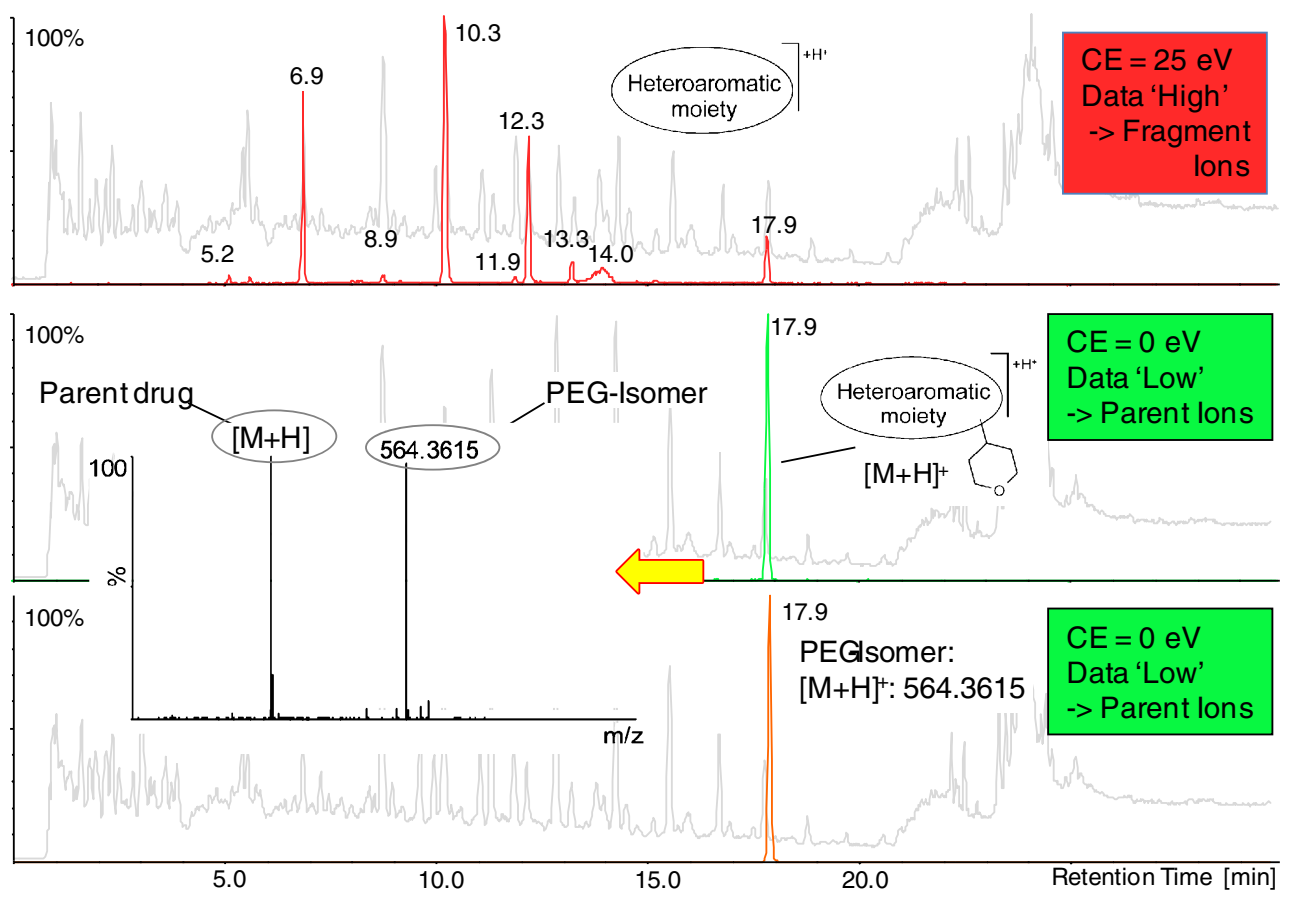


\section{UPLC/TWIMS/MS ${ }^{\mathrm{E}}$ QTOF mass spectrometry}

Metabolite profiles of the sample material were analyzed by ultra high performance liquid chromatography coupled to traveling wave ion mobility separation QTOF mass spectrometry using the $\mathrm{MS}^{\mathrm{E}}$ acquisition methodology. Volumes of $5 \mu \mathrm{l}$ of sample material were injected on column. The UPLCsystem (Acquity UPLC, Sample Manager 2777, Binary solvent manager, Column Heater/Cooler, all Waters, Milford, MA, USA) was coupled to a Synapt G2 mass spectrometer (Waters MS Technologies, Manchester, UK). Samples were analyzed by reverse phase UPLC using $150 \times 3.0 \mathrm{~mm}$ columns (Ascentis express Phenyl-Hexyl, $2.7 \mu \mathrm{m}$, Supelco, USA) with a gradient of aqueous ammonium formate $(0.05 \mathrm{M}, \mathrm{pH} 8)$ as eluent A versus acetonitrile/methanol (1:1) as eluent B with a flow rate of $0.6 \mathrm{ml} / \mathrm{min}$ (gradient: $5 \% \mathrm{~B}$ at $0 \mathrm{~min}, 5 \% \mathrm{~B}$ at

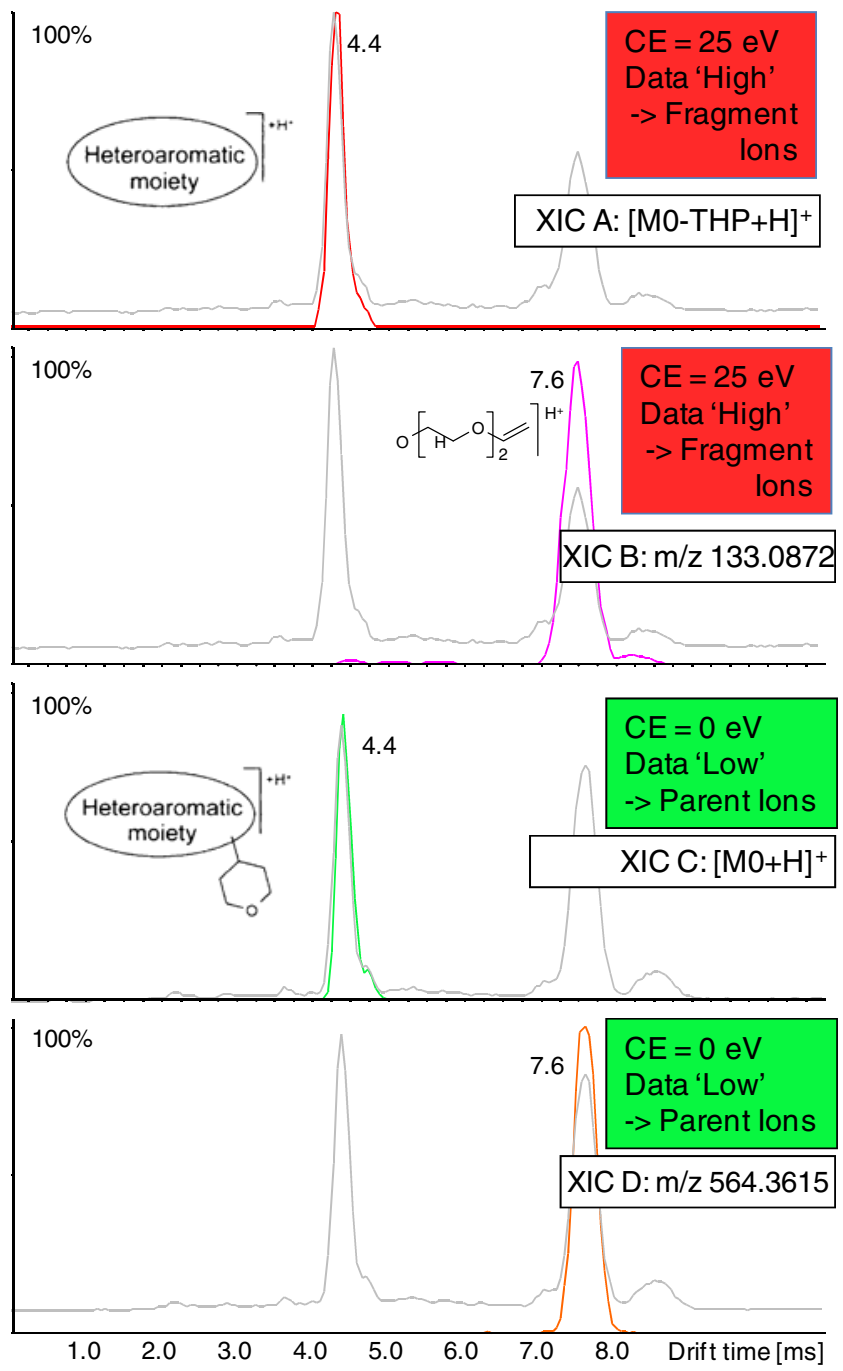

Fig. 6 Urine after administration of M0. Mass data 'high' and 'low' at $\mathrm{rt}=17.9 \mathrm{~min} \pm 1.5 \mathrm{~s}$, plotted versus the drift time. Upper 2 traces: data 'high': $\mathrm{XIC}$ of $[\mathrm{M}-\mathrm{THP}+\mathrm{H}]^{+}$, and $\mathrm{PEG}$ fragment, light gray: TIC Lower 2 traces: data 'low': XIC of parent drug's and PEG's $[\mathrm{M}+\mathrm{H}]^{+}$, light gray: TIC
$1 \mathrm{~min}, 30 \% \mathrm{~B}$ at $20 \mathrm{~min}, 99 \% \mathrm{~B}$ at $23 \mathrm{~min}, 99 \% \mathrm{~B}$ at $32 \mathrm{~min}$ ). The column temperature was $40{ }^{\circ} \mathrm{C}$.

The combined Z-spray/lockspray interface of the Synapt G2 $[38,39]$ was operated in positive ion mode with nitrogen as cone and desolvation gas at flow rates of $20 \mathrm{~L} / \mathrm{h}$ and $1,000 \mathrm{~L} / \mathrm{h}$, respectively. The source temperature and desolvation temperature were set to $120^{\circ} \mathrm{C}$ and $550{ }^{\circ} \mathrm{C}$, respectively. The capillary, sampling cone and extraction cone voltages were set to $3.0 \mathrm{kV}, 20 \mathrm{~V}$ and $4 \mathrm{~V}$, respectively. The first quadrupole operated in non-resolving RF-only mode. The collision energy of the trap cell was set to $4 \mathrm{eV}$. Mass spectral data over the range $\mathrm{m} / \mathrm{z}$ 50-1200 were acquired in two separate channels using two continuously interlaced collision energies of the transfer cell, at $0 \mathrm{eV}$ and $25 \mathrm{eV}$, with a switching frequency of $2 \mathrm{~Hz}$. The trap, IMS and transfer traveling wave devices were operated with traveling wave amplitudes and velocities of $8.0 \mathrm{~V}$ and $508 \mathrm{~m} / \mathrm{s}, 40.0 \mathrm{~V}$ and $1000 \mathrm{~m} / \mathrm{s}$, and $3.0 \mathrm{~V}$ and $450 \mathrm{~m} / \mathrm{s}$, respectively. The trap/transfer cells were operated with argon at a pressure of $2.5 \mathrm{e}-2$ mbar. Nitrogen as drift gas was introduced into the IMS cell to maintain a pressure of 3.1 mbar. The time of flight analyzer was operated using $\mathrm{V}$-mode optics with an average mass resolution of $\mathrm{m} / \Delta \mathrm{m}=$ 20,000 (full-width at half-maximum definition). The TOFpusher and trap-gate frequencies were $18.5 \mathrm{kHz}$ and $92.5 \mathrm{~Hz}$, respectively. Thus, individual IMS cycles of $10.8 \mathrm{~ms}$ duration were analyzed with a pusher period of $54 \mu$ s resulting in 200 TOF pushes/IMS cycle. Mass data were lock mass calibrated with leucine-enkephalin $(\mathrm{m} / \mathrm{z}$ 556.2771). The calibrant was introduced with a flow rate of $10 \mu \mathrm{l} / \mathrm{min}$ and was measured for $0.5 \mathrm{~s}$ with a frequency of $1 /$ min over the full chromatographic run time. Lock mass data were acquired in a third separate channel. Data acquisition and processing were performed using Masslynx (V4.1, SCN714), Driftscope (V2.1) and $\mathrm{MS}^{\mathrm{E}}$ viewer (V1.2).

\section{Results and discussion}

Pooled samples of plasma, urine, bile and faeces after oral administration of a drug compound to rats were investigated in this study. Native urine and bile were diluted; plasma and faeces were extracted before analysis.

The investigated compound (M0), a tetrahydropyranyl (THP) derivative with substituted heteroaromatic system, was subject to extensive phase I metabolism (Fig. 1). Metabolites were formed by initial oxidation of the THP-moiety in 2-, 3- and 4-position (metabolite fraction M8). Two major metabolites, the ring-open carboxylic acid/alcohol (M1) and the diol (M2), were formed by initial oxidation in 2-position of the THP-moiety followed by further oxidation (leading to M1) or reduction (M2), respectively. Adducts with urea were identified in urine (M3, M4). Minor metabolites were 
formed by oxidation of the heteroaromatic system (M5, M6, M7). Phase II transformations played a subordinate role.

Basic principles of metabolite screening by UPLC/TWIMS/MS ${ }^{\text {E }}$ QTOF-MS

In general, samples were analyzed for drug related compounds by two independent, orthogonal separation techniques including UPLC (ultra high performance liquid chromatography) and TWIMS (travelling wave ion mobility separation) followed by detection with high resolution QTOF mass spectrometry using the $\mathrm{MS}^{\mathrm{E}}$ acquisition approach. The instrument was operated in positive ion mode with the first quadrupole operating non-resolving, in RFonly mode.

The triwave geometry of the instruments ion mobility cell $[38,39]$ allows fragmentation in both the trap (pre IMS separation) and the transfer regions (post IMS separation). In this application, transfer fragmentation was performed. According to the $\mathrm{MS}^{\mathrm{E}}$ acquisition methodology the collision energy was alternately switched with a frequency of $2 \mathrm{~Hz}$ between two settings 'low $(=0 \mathrm{eV})$ ' and 'high $(=25 \mathrm{eV})$ ' which allowed the parallel detection of $[\mathrm{M}+\mathrm{H}]^{+}$-ions with only minimal fragmentation and of only fragment ions, respectively (Fig. 2). [M $+\mathrm{H}]^{+}$-ions and fragments of all, endogenous compounds and drug related compounds of interest, were acquired in two separated data sets 'low' and 'high', respectively. Ions from an individual compound e.g. $[\mathrm{M}+\mathrm{H}]^{+}$-ions in the data 'low' and their specific fragments in the data 'high', are aligned by the retention time (retention time alignment).

In combination with ion mobility separation, $[\mathrm{M}+\mathrm{H}]^{+}$ions from the RF-only quadrupole are first collected in the trap cell of the instruments triwave device and then periodically released in packages into the IMS cell (Fig. 2). After separation due to differences in collisional cross sections, they are transported into the transfer cell. Here, with interlaced collision energies, the $[\mathrm{M}+\mathrm{H}]^{+}$-ions were either transported with a low collision energy $(0 \mathrm{eV})$ without or minimum fragmentation to the TOF detector or subjected to additional fragmentation with a higher collision energy $(25 \mathrm{eV})$. With collision energy applied after ion mobility separation, fragments retain the original drift time of the parent ions. As a result, all ions of an individual compound e.g. $[\mathrm{M}+\mathrm{H}]^{+}$, and its fragments are aligned not only by retention time, but additionally by drift time (=retention time and drift time alignment).

In summary, all ion species of the two data sets 'high' (product ions) and 'low' (parent ions) were characterized by the four dimensions intensity $(\mathrm{I})$, mass $(\mathrm{m} / \mathrm{z})$, retention time (rt) and drift time (dt). Ions of individual compounds are aligned by specific combinations of retention- and drift times.

\section{Data evaluation, basic principles}

The general concept of screening for metabolites by diagnostic drug related fragments by $\mathrm{rt} / \mathrm{dt}$-aligned $\mathrm{MS}^{\mathrm{E}}$ data is discussed in the following:

The basis for the metabolite screening with the $\mathrm{MS}^{\mathrm{E}}$ methodology is a sound understanding of the fragmentation pathways of the parent drug's $[\mathrm{M}+\mathrm{H}]^{+}$. Drug related compounds can be identified by extracted ion chromatograms (XICs) of diagnostic fragments in the high collision energy data ('data high') which are specific for defined substructures
Fig. 7 Urine after administration of M0. Mass data at $\mathrm{rt}=17.9 \mathrm{~min}$ before and after mobility separation (rt/dt-windows: $1.5 \mathrm{~s} / 1.5 \mathrm{~ms}$ ). Left: MS-spectra of data 'low', drift time separation of

$[\mathrm{M}+\mathrm{H}]^{+}$-parent ions at 4.4 and 7.6 ms. Right: MS/MS-spectra of data 'high', remaining $[\mathrm{M}+\mathrm{H}]^{+}$- and fragment ions with conserved drift time separation of $[\mathrm{M}+\mathrm{H}]^{+}$parent ions
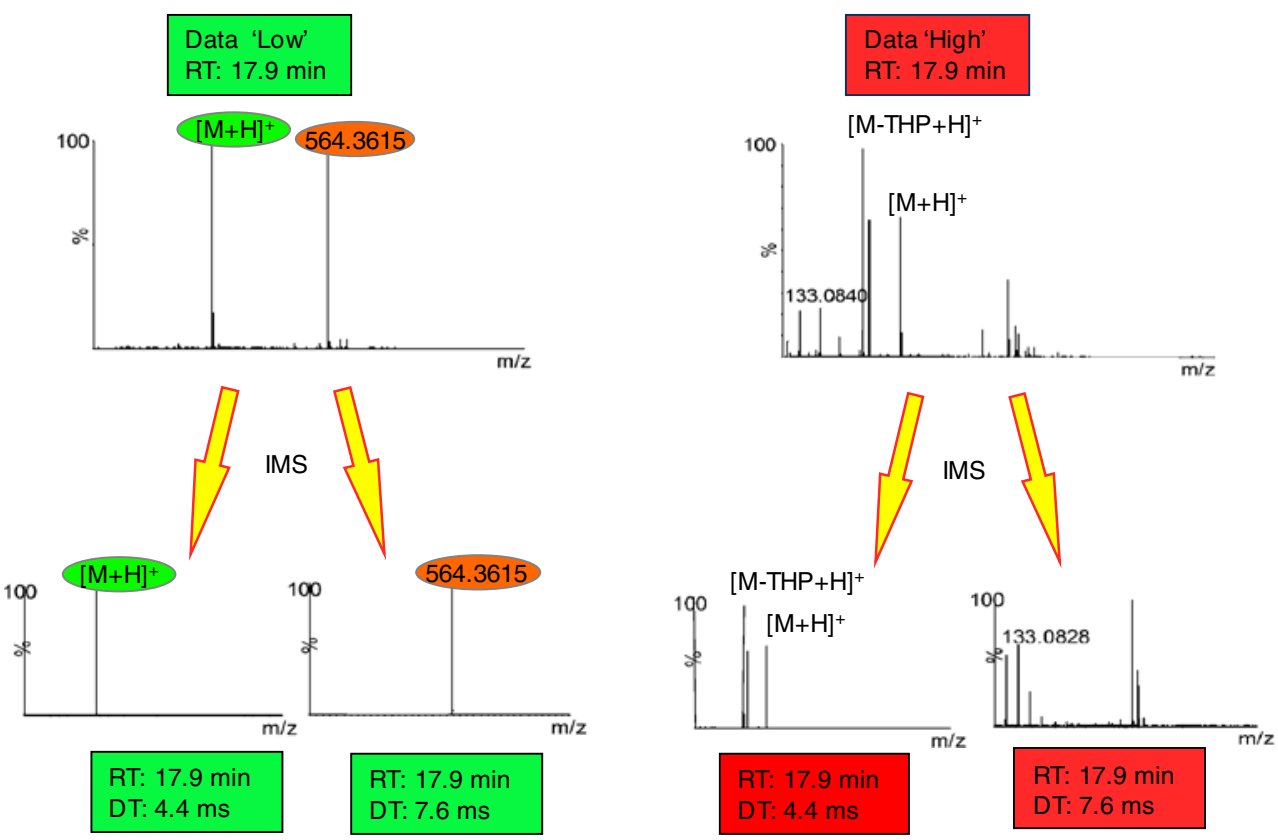
of the parent compound. Associated $[\mathrm{M}+\mathrm{H}]^{+}$-ions are identified with their XICs in the low collision energy data ('data low') by correlation with exactly matching retention times and chromatographic profiles and drift times and vice versa. Independent of the intensity, any known fragment or - with respect to putative biotransformations - any expected product ion can be used for this approach. This methodology is presented in the following sections.

The product ion spectrum of the parent drug's $[\mathrm{M}+\mathrm{H}]^{+}$, M0, is shown in Fig. 3. The base ion was formed by loss of the THP-residue, $[\mathrm{M}-\mathrm{THP}+\mathrm{H}]^{+}$, and was used as diagnostic fragment for metabolites with unchanged heterocyclic moiety. In Fig. 4 the search for metabolites with $[\mathrm{M}-\mathrm{THP}+\mathrm{H}]^{+}$ in urine is presented. The extracted ion chromatogram of $[\mathrm{M}-\mathrm{THP}+\mathrm{H}]^{+}$in the data 'high' is displayed in the upper trace (Fig. 4, XIC A). The chromatograms below show the XICs of the correlated $[\mathrm{M}+\mathrm{H}]^{+}$-ions of the parent compound M0 (XIC B) and the metabolites M1 (XIC C: M0+2O), M2 (XIC D: $\mathrm{M} 0+\mathrm{O}+2 \mathrm{H}$ ), M3, M4 (XIC E: $\mathrm{M} 0+\mathrm{CH}_{2} \mathrm{~N}_{2} \mathrm{O}$ ) and
M8 (XIC F: M0+O) in the data 'low'. Metabolite specific ions of both data 'high' and 'low' exhibit exactly the same peak shape. The higher the chromatographic resolution, the better the alignment of metabolite related peaks and the discrimination of matrix signals.

With sample material of in vivo studies a complete separation of interferences is only achieved in rare cases due to the high matrix burden. Thus, the unambiguous structure assignment based on only retention time aligned 'high' and 'low' data alone is challenging, sometimes impossible. With the introduction of the additional dimension of separation by ion mobility, completely purified MS- and MS/MS data are achievable. In Fig. 5 a common situation of a strong interference with matrix compounds with nearly identical chromatographic profile to the compound of interest is shown. The parent compound, M0, co eluted with a polyethylenglycol isomer, $[\mathrm{M}+\mathrm{H}]^{+} \mathrm{m} / \mathrm{z} 564$, at the retention time of $17.9 \mathrm{~min}$. The IMS data can be used to unambiguously resolve these
Fig. 8 Urine after administration of M0. Mass data 'low' at retention time of metabolite fraction M8 at $\mathrm{rt}=14.0 \mathrm{~min} \pm 1.5 \mathrm{~s}$. Upper spectrum: Mass data over all drift times: 0-10 ms. Lower spectra: Mass data of metabolite ions and prominent matrix signals at defined drift time windows $( \pm 1.5 \mathrm{~ms})$

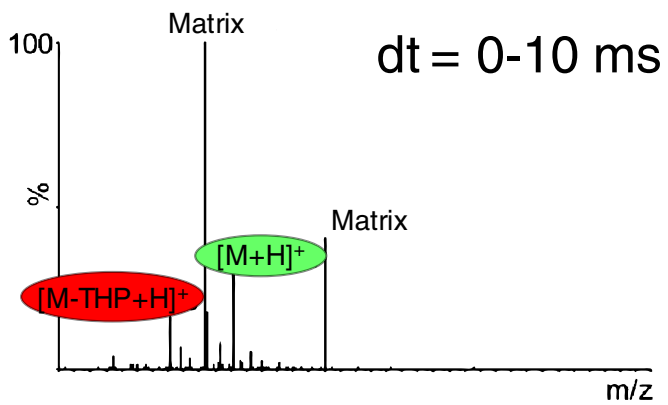

\section{Matrix:}
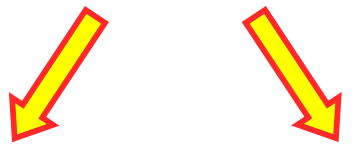

\section{Drug related:}
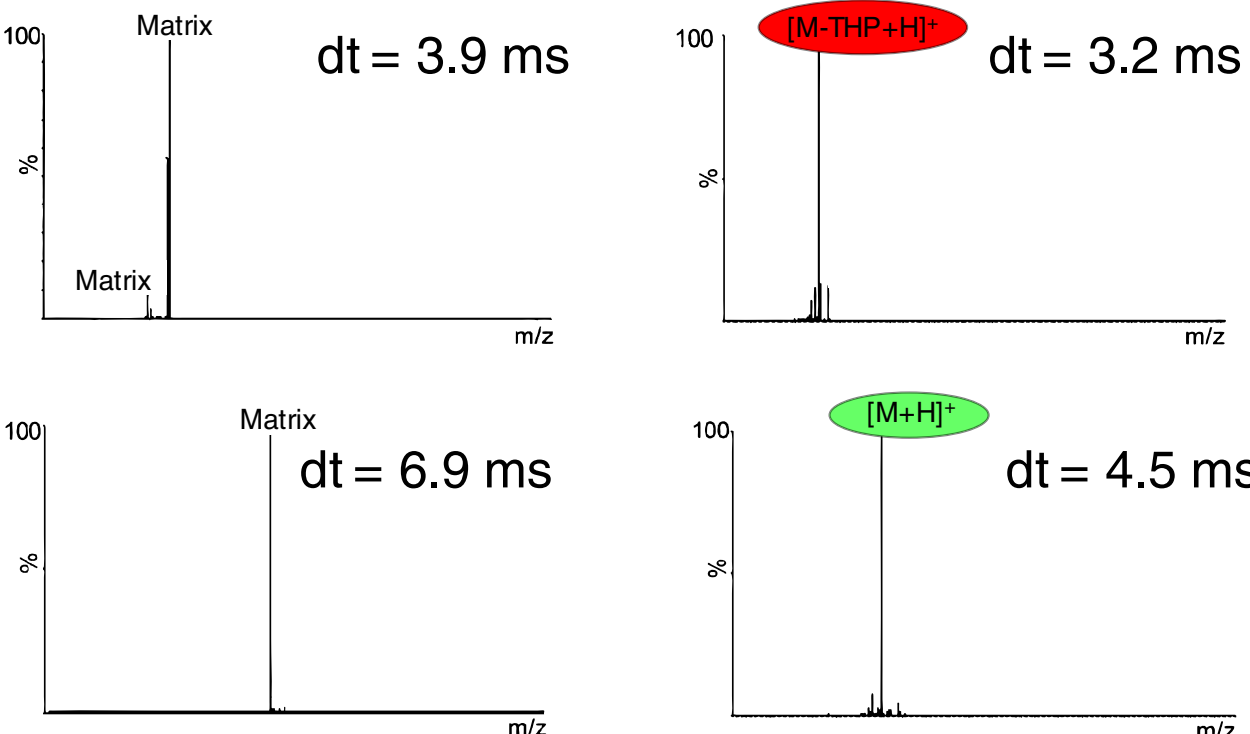


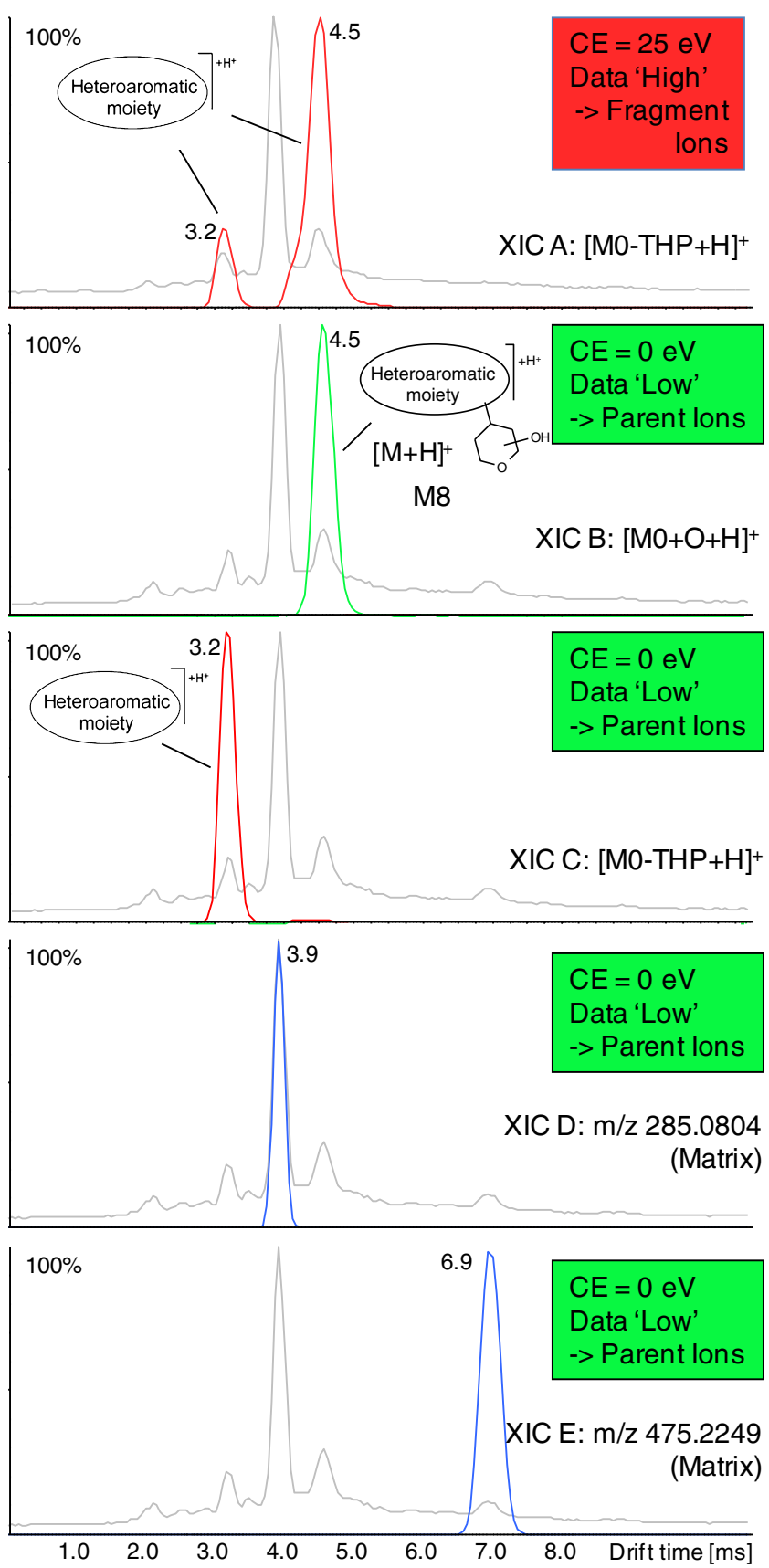

Fig. 9 Urine after administration of M0. M8-data 'high' and 'low' at $\mathrm{rt}=14.0 \min \pm 1.5 \mathrm{~s}$, plotted versus the drift time. Upper trace: data 'high': XIC of the diagnostic $[\mathrm{M}-\mathrm{THP}+\mathrm{H}]^{+}$-ion, light gray: TIC Lower traces: data 'low': XICs of the $[\mathrm{M}+\mathrm{H}]^{+}$-ion of M8 and its in-source fragment $[\mathrm{M}-\mathrm{THP}+\mathrm{H}]^{+}$together with XICs of additional matrix signals with significant contribution to the TIC, light gray: TIC

peaks and align their fragments without the need for dedicated MS/MS investigations. Figure 6 shows the XIC data 'high' (2 upper traces) and 'low' (2 lower traces) of the peak top at 17.9 min (average, $\pm 1.5 \mathrm{~s}$ ) which is now plotted versus the drift time dimension. Again, the XIC of the diagnostic fragment, $[\mathrm{M} 0-\mathrm{THP}+\mathrm{H}]^{+}$ (XIC A) can be used as indicator for the drug related compounds. The drift time for this fragment was $4.4 \mathrm{~ms}$. The aligned $[\mathrm{M}+\mathrm{H}]^{+}$of the parent compound was unambiguously identified by the corresponding XIC in the data 'low' (XIC C). The $[\mathrm{M}+\mathrm{H}]^{+}$of the PEG-isomer, separated by IMS was detected with a drift time of $7.6 \mathrm{~ms}$ (XIC D). Its fragment $\mathrm{C}_{6} \mathrm{H}_{13} \mathrm{O}_{3}{ }^{+}, \mathrm{m} / \mathrm{z} 133$, which, in a similar manner, can be used as general diagnostic fragment for PEG-isomers (see below) co eluted with the same drift time at $7.6 \mathrm{~ms}$ (XIC B).

Figure 7 demonstrates the improvement of the data quality of MS- (data 'low', left) and MS/MS-spectra (data 'high', right) from rt-aligned data to combined rt/ dt-aligned data. The $[\mathrm{M}+\mathrm{H}]^{+}$-ion species of the parent drug and the PEG-isomer were separated from one another and were additionally completely stripped of other interferences by applying appropriate retention time and drift time windows of $\pm 1.5 \mathrm{~s}$ and $\pm 1.5 \mathrm{~ms}$, respectively. Due to the conservation of the $[\mathrm{M}+\mathrm{H}]^{+}$parent ion drift times, the parent compound- and PEGfragments were also completely separated and cleaned from one another and clear of other matrix interferents. Thus, the combined rt/dt-aligned MS- and MS/MS-spectra can directly be used for structure elucidation without compromise.

A second representative met-ID example is presented using the metabolite fraction M8 which consisted of oxidation products of the THP moiety. The XIC of the $[\mathrm{M}+\mathrm{H}]^{+}$ showed a broad peak at 14.0 min (see Fig. 4, XIC F). In Fig. 8, the mass spectra of the data 'low' of the peak top at $14.0 \mathrm{~min}( \pm 1.5 \mathrm{~s})$ without and with ion mobility separation is presented: Without IMS the $[\mathrm{M}+\mathrm{H}]^{+}$together with its in source fragment $[\mathrm{M}-\mathrm{THP}+\mathrm{H}]^{+}$were present with high abundant interferences of co eluting matrix compounds (upper spectrum). The ions were separated and aligned by IMS into drug related ions $(\mathrm{dt}=3.2 \mathrm{~ms}$ and $4.5 \mathrm{~ms})$ and matrix signals $(\mathrm{dt}=3.9 \mathrm{~ms}$ and $6.9 \mathrm{~ms})$. The spectra are shown with $\mathrm{rt} / \mathrm{dt}-$ windows of $\pm 1.5 \mathrm{~s} / \pm 1.5 \mathrm{~ms}$. The process of ion alignment is demonstrated again in Fig. 9: Here the 'high' and 'low' mass data of the peak top at $14.0 \mathrm{~min}( \pm 1.5 \mathrm{~s})$ are plotted versus the drift time; XICs which significantly contributed to the total ion current are selected. The XIC of the diagnostic fragment, $[\mathrm{M}-\mathrm{THP}+\mathrm{H}]^{+}$of the data 'high' showed two peaks at the drift times $3.2 \mathrm{~ms}$ and $4.5 \mathrm{~ms}$ (upper trace, XIC A). In the data 'low' the XIC of the M8's $[\mathrm{M}+\mathrm{H}]^{+}$(at $4.5 \mathrm{~ms}$, XIC B) could be unambiguously aligned with the diagnostic fragment of the data 'high'. In this example, the second peak at $3.2 \mathrm{~ms}$ (XIC C) corresponded to the XIC of the $[\mathrm{M}-\mathrm{THP}+\mathrm{H}]^{+}$itself, which was formed by in-source fragmentation and therefore resolved in the IMS cell from the parent ion prior to the transfer cell fragmentation process. The prominent matrix ions were completely separated with drift times of $3.9 \mathrm{~ms}$ and $6.9 \mathrm{~ms}$, respectively (XIC D and E). 


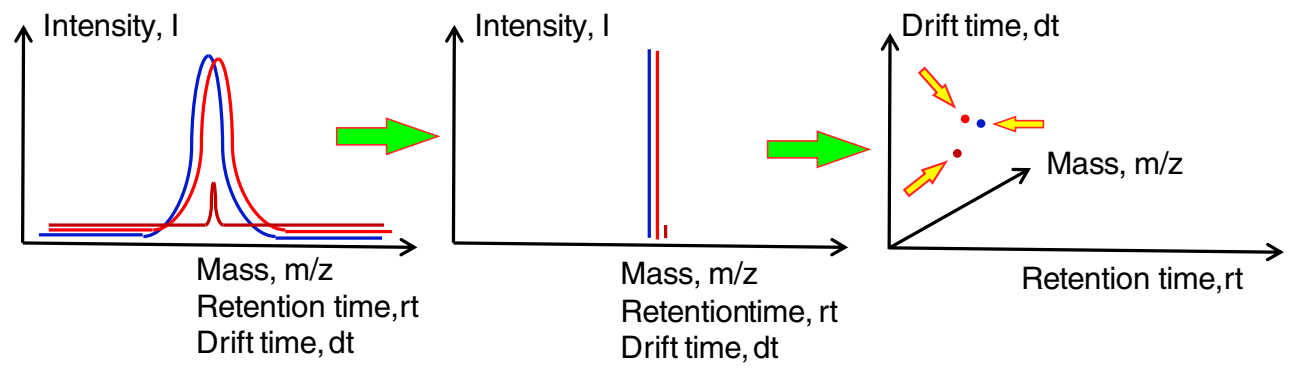

Fig. 10 4D Apex convolution principle: Left picture: continuum 4D raw data which is defined by intensity $(\mathrm{I})$, mass $(\mathrm{m} / \mathrm{z})$, retention time $(\mathrm{rt})$, and drift time (dt). Mid picture: continuum data is convoluted to

Data evaluation, implementation of automated data mining tools

The general approach of the data evaluation process as discussed in the previous section is laborious and time consuming. discrete components. Right picture: display of convoluted data in the $\mathrm{m} / \mathrm{z}$-/rt-/dt-data space: signals are separated when differentiated in at least one of the dimensions $\mathrm{m} / \mathrm{z}$, rt or $\mathrm{dt}$

For an accelerated routine analysis of the 4D 'high' and 'low' data the $\mathrm{MS}^{\mathrm{E}}$ viewer was used as an automated software tool. In a first step of data processing, the included apex convolution algorithm reduced the raw data, i.e. the continuum data with respect to the dimension, mass $(\mathrm{m} / \mathrm{z})$, retention time (rt) and
Fig. 11 Identification of drug related compound in rat faeces after 4D apex convolution of 'high' and 'low' data: $\mathrm{rt} / \mathrm{dt}$-alignment of component signals (rt/dt-windows: \pm 1.5 $\mathrm{s} / \pm 0.05 \mathrm{~ms})$. Upper trace: $[\mathrm{M}-\mathrm{THP}+\mathrm{H}]^{+}$component peaks versus retention time. Lower traces: parent drug's and metabolite's $[\mathrm{M}+\mathrm{H}]^{+}$ component peaks after rt/dtalignment versus retention time

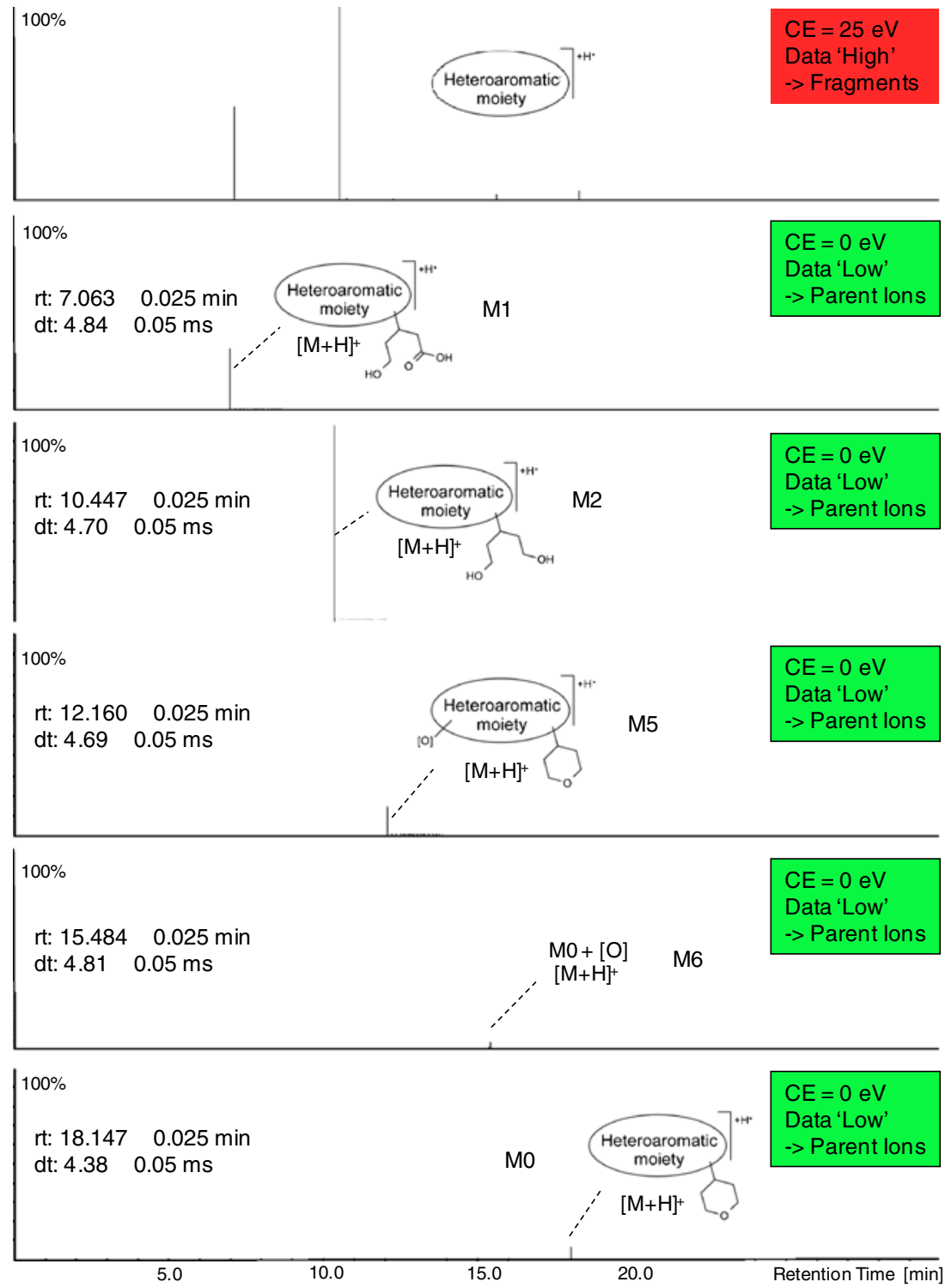


Fig. 12 Metabolite M1 in faeces (rt: $7.06 \mathrm{~min}$ ), Data 'low': a continuum data, b after apex convolution and rt/dtalignment (rt: $18.147 \pm 1.5 \mathrm{~s}$, dt: $4.38 \pm 0.05 \mathrm{~ms}$ ). Data 'high': c continuum data, $\mathbf{d}$ after apex convolution and $\mathrm{rt} / \mathrm{dt}$-alignment (rt: $18.147 \pm 1.5 \mathrm{~s}, \mathrm{dt}$ : $4.38 \pm 0.05 \mathrm{~ms}$ )
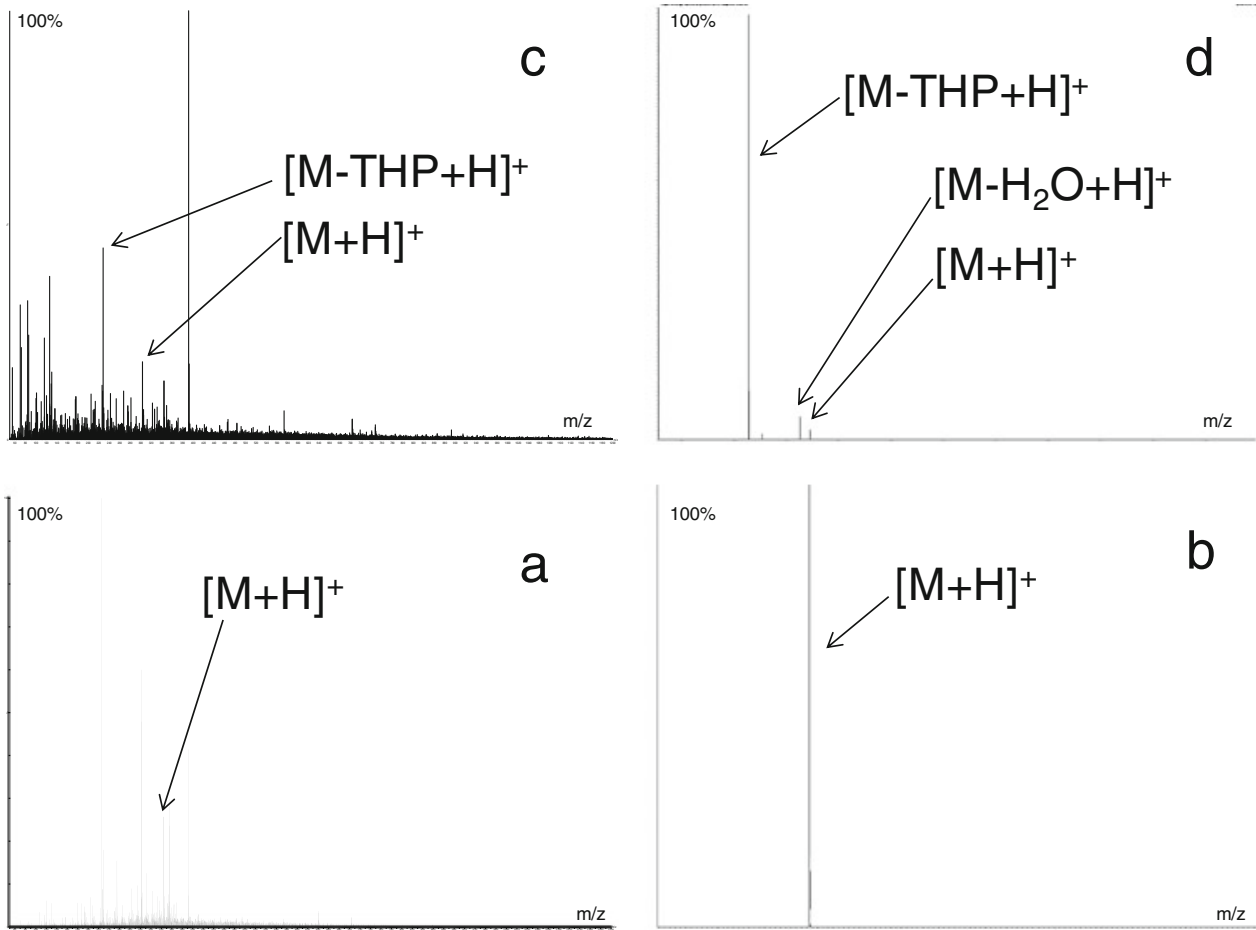

drift time (dt), to single discrete component data (Fig. 10). Consequently, a particular ion of interest can be displayed as a single data point in a 3 dimensional data space which is defined by the axes $\mathrm{m} / \mathrm{z}, \mathrm{rt}$, and $\mathrm{dt}$. In this $3 \mathrm{D}$ data space, signals are resolved which are differentiated in at least one of the parameter $\mathrm{m} / \mathrm{z}$, rt or $\mathrm{dt}$. The fourth dimension which is the intensity (I) is not displayed in Fig. 10. In the second step of data processing, the $\mathrm{MS}^{\mathrm{E}}$ viewer allows the extraction of purified MS- and MS/MS data by appropriate settings of retention time and drift time windows. The drift time is implemented as a filter and the display can be toggled to show the data with and without dt-separation.

With regard to the worst case of in vivo met-ID studies, the very low abundance metabolite signals are resolved in
Fig. 13 Metabolite M6 in faeces (rt: $12.16 \mathrm{~min}$ ), Data 'low': a continuum data, b after apex convolution and $\mathrm{rt} / \mathrm{dt}-$ alignment (rt: $18.147 \pm 1.5 \mathrm{~s}$, dt: $4.38 \pm 0.05 \mathrm{~ms})$. Data 'high': c continuum data, $\mathbf{d}$ after apex convolution and $\mathrm{rt} / \mathrm{dt}$-alignment (rt: $18.147 \pm 1.5 \mathrm{~s}, \mathrm{dt}$ :

$4.38 \pm 0.05 \mathrm{~ms})$
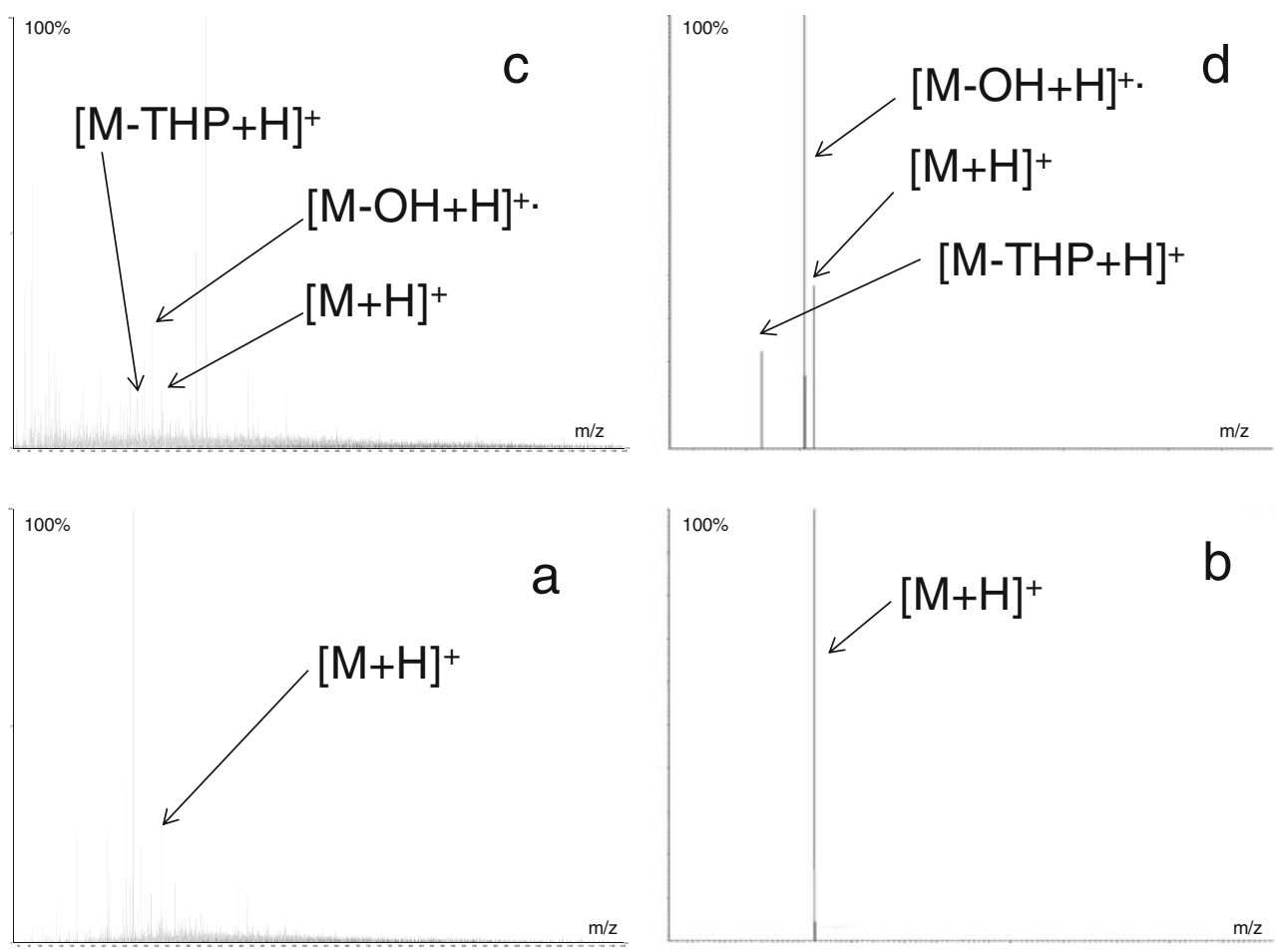
the $3 \mathrm{D}$-data space $(\mathrm{m} / \mathrm{z}$ vs. rt vs. $\mathrm{dt})$ and can be identified as drug related signals, even in the presence of very intense co eluting matrix ions.

The above process is demonstrated in the following, using a common challenging, in vivo met-ID example: the analysis of an extract of rat faeces with low concentrations of drug related compounds together with very high matrix burden.

The continuum raw data 'high' and 'low' were converted to discrete component data by the peak detection software for further metabolite analysis. In Fig. 11 the screening for drug related compounds in the faeces sample with the diagnostic $[\mathrm{M}-\mathrm{THP}+\mathrm{H}]^{+}$fragment is presented: The upper trace shows the 'XIC' of the $[\mathrm{M}-\mathrm{THP}+\mathrm{H}]^{+}$of the peak detected component data 'high'. The lower traces shows the automatically rt/dt-aligned $[\mathrm{M}+\mathrm{H}]^{+}$-ions of the parent drug (M0) and the metabolites M1, M2, M5 and M6 of the peak detected component data 'low'. Due to the orthogonality of UPLC and TWIMS, interfering ions of unwanted matrix are efficiently discriminated. The spectra are shown with rtand dt-windows of $\pm 1.5 \mathrm{~s}$ and $\pm 0.05 \mathrm{~ms}$, respectively.

In the Figs. 12 and 13 the comparison of continuum raw data at the peak tops of the $[\mathrm{M}+\mathrm{H}]^{+}$-XICs of the metabolites M1 and M6 versus the rt/dt-aligned MS data is presented. The continuum 'high' and 'low' raw data (Figs. 12 and 13, left) demonstrate the analytical challenge with low abundant analyte signals and high matrix interferences which is a common situation with in vivo sample material, especially in samples of faeces or bile. After apex convolution in combination with rt/dt-alignment the MS- and MS/MSspectra (Figs. 12 and 13, right) were completely purified from all matrix signals. The extracted data were identical to directed MS/MS-spectra of selected $[\mathrm{M}+\mathrm{H}]^{+}$-parent ions and can be used without compromises for structure elucidation and further downstream processes. The advantage over directed analytical approaches is the simplified setup which is completely unbiased: Without compromise, rt/dt-aligned MS- and MS/MS-data from any compound of interest can be extracted retrospectively at any time. The shown examples of the metabolites M1 and M6 are representative for all analytes including M0, M2, M3, M5. The rt/dtalignment resulted to cleaned MS- and MS/MS spectra (data not shown here).

To further demonstrate the universality of this approach an excursus to the analysis of the PEG-isomers is presented. As discussed earlier the parent compound co eluted with a PEG-isomer with nearly the same retention time at $17.9 \mathrm{~min}$ (Fig. 5). Figure 7 showed the alignment of the $[\mathrm{M}+\mathrm{H}]^{+}, \mathrm{m} / \mathrm{z}$ 564 (data 'low'), of the PEG-isomer with $\mathrm{m} / \mathrm{z} 133.0865$ $\left(\mathrm{C}_{6} \mathrm{H}_{13} \mathrm{O}_{3}^{+}\right)$of the data 'high', which is a common fragment of this compound class [40]. This general PEG-related fragment can be used as diagnostic ion for PEG-isomers in the original data 'high' and 'low' for the identification of all PEG-isomers which were present in the urine sample (Fig. 14): The XIC of $\mathrm{m} / \mathrm{z} 133.0865$ is shown in the upper trace; a superimposition of aligned $[\mathrm{M}+\mathrm{H}]^{+}$-XICs of the PEG-isomers was correlated in the lower trace. Data processing with the $\mathrm{MS}^{\mathrm{E}}$ viewer by apex convolution and rt/dtalignment resulted also for this PEG-isomers to completely purified MS- and MS/MS-data for further structure elucidation/assignment (data not presented).

\section{Summary and conclusions}

This new approach for the screening and elucidation of drugs and their metabolites in complex matrix consists of the combination of two orthogonal separation techniques, UPLC (ultra high performance liquid chromatography) and TWIMS (traveling wave ion mobility separation), together with high resolution QTOF mass spectrometry using the $\mathrm{MS}^{\mathrm{E}}$ acquisition methodology. Two mass data sets were acquired separately in two channels using interlaced collision energies detecting $[\mathrm{M}+\mathrm{H}]^{+}$parent ions and product ions in parallel. All ion species of the two data sets were characterized by the four dimensions intensity (I), mass $(\mathrm{m} / \mathrm{z})$, retention time (rt) and drift time (dt). The raw data were
Fig. 14 Urine after administration of M0. Data 'high'and 'low'. Upper trace: data 'high': XIC of PEG fragment, $\mathrm{m} / \mathrm{z} 133$, light gray: TIC. Lower trace: data 'low': superimposition XICs of PEG-isomers $[\mathrm{M}+\mathrm{H}]^{+}$, light gray: TIC

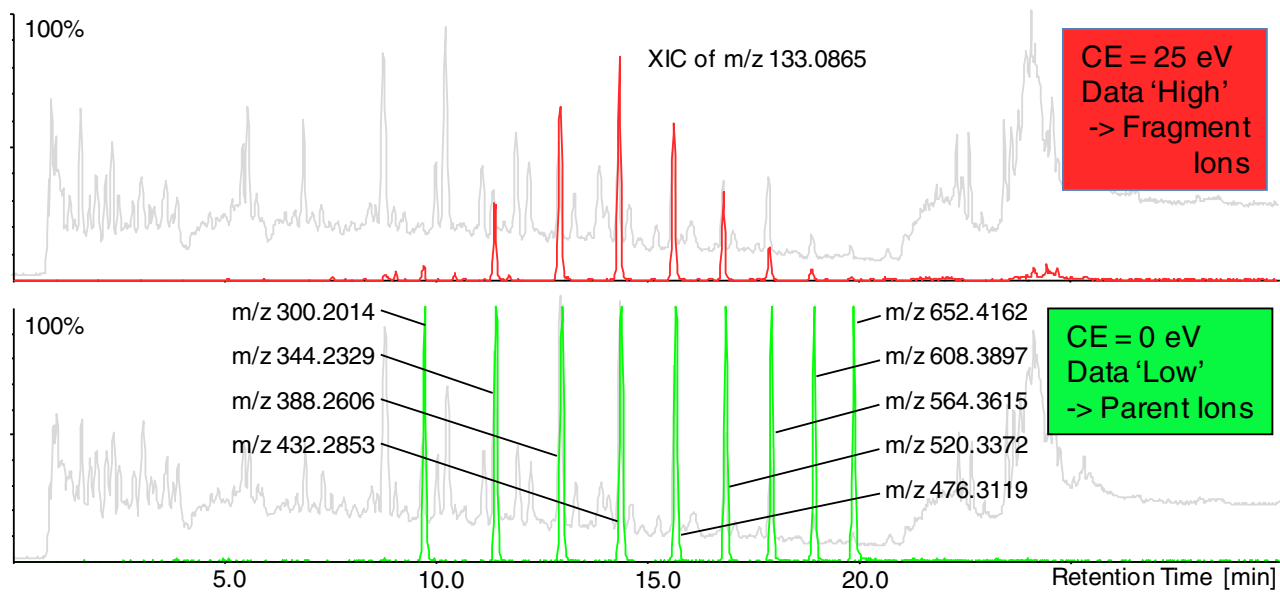


processed by the apex convolution software for the reduction of the continuum m/z-, rt- and dt-data to discrete components. As a result, ions that differ in at least one of the dimensions $\mathrm{m} / \mathrm{z}$, rt or $\mathrm{dt}$ were resolved. Using fragmentation after ion mobility separation, $[\mathrm{M}+\mathrm{H}]^{+}$ion species of the low collision energy channel are aligned with their related fragments of the high collision energy channel by virtue of possessing the same combination of retention time and drift time. Thus, associated MS- and MS/MS-data for compounds of interest are extractable with appropriate rt- and dt-windows. Due to the orthogonality of UPLC and TWIMS, unwanted interfering ions, e.g. high intensity matrix signals which are generally common in mass data of in vivo sample material, were efficiently discriminated. As result, completely purified MS- and MS/MS-data for any compounds can be extracted from the mass data for further evaluation. This principle supersedes the data dependent scan methodologies which are laborious and difficult to set up in complex sample materials with low concentrations of analytes and high matrix burden. The $\mathrm{MS}^{\mathrm{E}}$ viewer as a tailored software tool was used for automated data mining.

This contribution refers to the application of UPLC/ TWIMS/MS ${ }^{\text {E }}$ QTOF Mass Spectrometry to drug metabolism studies. Its general and universal applicability is demonstrated by the analysis of in vivo sample material of especially faeces containing highest concentrations of matrix components and low concentrations of analytes. The MS- and MS/MS data of drug related compounds was completely purified from matrix signals and could be used for further processing, e.g. structure elucidation/structure assignment, without compromise.

In general the unbiased 'high/low' 4D data sets open the field for retrospective analysis of any compound at any time including drug metabolites, up and down regulated endogenous compounds, small molecules and proteins without the need for sample reanalysis which is always questionable with respect to stability and storage conditions. Thus, the $4 \mathrm{D}$ data sets can be used as comprehensive data repositories during the development process of a drug including early in vitro investigations, first in man trials, drug metabolism studies in samples of toxicology species and late stage ${ }^{14} \mathrm{C}$ human ADME trials. This generic approach is particularly useful in cases where the coverage of human metabolites need to be retrospectively demonstrated in previously analyzed preclinical samples of toxicology testing.

\footnotetext{
Acknowledgements The authors thank the non-clinical pharmacokinetics group, BI Pharma GmbH \& Co. KG, Germany, for conducting the animal studies and providing the sample material. We are grateful to Thomas Ebner and Uli Roth for their support. Additionally, we would like to thank Richard Lock (Waters, Manchester, UK) and Mark Wrona (Waters, Milford, USA) for their excellent assistance and John Rontree (Waters, Manchester, UK) for infecting us with the TWIMS-technology.
}

Open Access This article is distributed under the terms of the Creative Commons Attribution License which permits any use, distribution, and reproduction in any medium, provided the original author(s) and the source are credited.

\section{References}

1. FDA, Food and Drug Administration, Center for Drug Evaluation and research (CDER), U,S. Department of Health and Human Services (2008) Safety testing of drug metabolites, Guidance for Industry, http://www.fda.gov/OHRMS/DOCKETS/98fr/FDA2008-D-0065-GDL.pdf

2. ICH, International conference on harmonization of technical requirements for registration of pharmaceuticals for human use (2009) ICH harmonized tripartite guideline, Guidance on nonclinical safety studies for the conduct of human clinical trials and marketing authorization for pharmaceuticals M3(R2) http:// www.ema.europa.eu/docs/en_GB/document_library/Scientific guideline/2009/09/WC500002720.pdf

3. Obach RS, Nedderman AN, Smith DA (2012) Radiolabelled massbalance excretion and metabolism studies in laboratory animals: are they still necessary? Xenobiotica 42:45-56

4. Clarke NJ, Rindgen D, Korfmacher WA, Cox KA (2001) Systematic LC/MS metabolite identification in drug discovery. Anal Chem 73:430A-439A

5. Li AC, Alton D, Bryant MS, Shou WZ (2005) Simultaneously quantifying parent drugs and screening for metabolites in plasma pharmacokinetic samples using selected reaction monitoring information-dependent acquisition on a QTrap instrument. Rapid Commun Mass Spectrom 19:1943-1950

6. Shou WZ, Magis L, Li AC, Naidong W, Bryant MS (2005) A novel approach to perform metabolite screening during the quantitative LC-MS/MS analyses of in vitro metabolic stability samples using a hybrid triple-quadrupole linear ion trap mass spectrometer. J Mass Spectrom 40:1347-1356

7. Li AC, Gohdes MA, Shou WZ (2007) 'N-in-one' strategy for metabolite identification using a liquid chromatography/hybrid triple quadrupole linear ion trap instrument using multiple dependent product ion scans triggered with full mass scan. Rapid Commun Mass Spectrom 21:1421-1430

8. Triolo A, Altamura M, Dimoulas T, Guidi A, Lecci A, Tramontana M (2005) In vivo metabolite detection and identification in drug discovery via LC-MS/MS with data-dependent scanning and postacquisition data mining. J Mass Spectrom 40:1572-1582

9. Peterman SM, Duczak N, Kalgutkar AS, Lame ME, Soglia JR (2006) Application of a linear ion trap/orbitrap mass spectrometer in metabolite characterization studies: examination of the human liver microsomal metabolism of the non-tricyclic anti-depressant nefazodone using data-dependent accurate mass measurements. J Am Soc Mass Spectrom 17:363-375

10. Li AC, Shou WZ, Mai TT, Jiang XY (2007) Complete profiling and characterization of in vitro nefazodone metabolites using two different tandem mass spectrometric platforms. Rapid Commun Mass Spectrom 21:4001-4008

11. Li AC, Shou WZ, Jiang XY (2009) Proposal of buspirone collision-induced dissociation rearrangement by exact mass measurements. Rapid Commun Mass Spectrom 23:1742-1745

12. Li AC, Ding J, Jiang X, Denissen J (2009) Two-injection workflow for a liquid chromatography/LTQ-Orbitrap system to complete in vivo biotransformation characterization: demonstration with buspirone metabolite identification. Rapid Commun Mass Spectrom 23:3003-3012 
13. Lim H-K, Chen J, Sensenhauser C, Cook K, Subrahmanyam V (2007) Metabolite identification by data-dependent accurate mass spectrometric analysis at resolving power of 60,000 in external calibration mode using an LTQ/Orbitrap. Rapid Commun Mass Spectrom 21:1821-1832

14. Lim H-K, Chen J, Cook K, Sensenhauser C, Silva J, Evans DC (2008) A generic method to detect electrophilic intermediates using isotopic pattern triggered data-dependent high-resolution accurate mass spectrometry. Rapid Commun Mass Spectrom 22:1295-1311

15. Ruan Q, Peterman S, Szewc MA, Ma L, Cui D, Humphreys WG, Zhu M (2008) An integrated method for metabolite detection and identification using a linear ion trap/Orbitrap mass spectrometer and multiple data processing techniques: application to indinavir metabolite detection. J Mass Spectrom 43:251-261

16. Zhu M, Ma L, Zhang H, Humphreys WG (2007) Detection and structural characterization of glutathione-trapped reactive metabolites using liquid chromatography-high-resolution mass spectrometry and mass defect filtering. Anal Chem 79:8333-8341

17. Zhu M, Ma L, Zhang D, Ray K, Zhao W, Humphreys WG, Skiles G, Sanders M, Zhanh H (2006) Detection and characterization of metabolites in biological matrices using mass defect filtering of liquid chromatography/high resolution mass spectrometry data. Drug Metab Dispos 34:1722-1733

18. Zhu M, Zhao W, Jimenez H, Zhanh D, Yeola S, Dai R, Vachharajani N, Mitroka J (2005) Cytochrome P450 3Amediated metabolism of buspirone in human liver microsomes. Drug Metab Dispos 33:500-507

19. Zhang D, Cheng PT, Zhang H (2007) Mass defect filtering on high resolution LC/MS data as a methodology for detecting metabolites with unpredictable structures: identification of oxazole-ring opened metabolites of muraglitazar. Drug Metab Lett 1:287-292

20. Zhang H, Zhang D, Ray KL (2003) A software filter to remove interference ions from drug metabolites in accurate mass liquid chromatography/mass spectrometric analyses. J Mass Spectrom 38:1110-1112

21. Zhang H, Zhu M, Rey KL, Ma L, Zhang D (2008) Mass defect profiles of biological matrices and the general applicability of mass defect filtering for metabolite detection. Rapid Commun Mass Spectrom 22:2082-2088

22. Cho R, Huang Y, Schwartz JC, Chen Y, Carlson TJ (2012) MS ${ }^{\mathrm{M}}$, an efficient workflow for metabolite identification using hybrid linear ion trap orbitrap mass spectrometer. J Am Soc Mass Spectrom 23:880-888

23. Wrona M, Mauriala T, Bateman KP, Mortishire-Smith RJ, O'Conner D (2005) 'All-in-one' analysis for metabolite identification using liquid chromatography/hybrid quadrupole time-offlight mass spectrometry with collision energy switching. Rapid Commun Mass Spectrom 19:2597-2602

24. Bateman KP, Castro-Perez J, Wrona M, Shockcor JP, Yu K, Oballa $\mathrm{R}$, Nicoll-Griffith DA (2007) $\mathrm{MS}^{\mathrm{E}}$ with mass defect filtering for in vitro and in vivo metabolite identification. Rapid Commun Mass Spectrom 21:1485-1496

25. Tiller PR, Yu S, Castro-Perez JM, Fillgrove KL, Baillie TA (2008) High-throughput, accurate mass liquid chromatography/tandem mass spectrometry on a quadrupole time-of-flight system as a 'first-line' approach for metabolite identification studies. Rapid Commun Mass Spectrom 22:1053-1061

26. Barbara JE, Castro-Perez JM (2011) High-resolution chromatography/time-of-flight $\mathrm{MS}^{\mathrm{E}}$ with in silico data mining is an information-rich approach to reactive metabolite screening. Rapid Commun Mass Spectrom 25:3029-3040

27. Plumb RS, Jones MD, Rainville P, Castro-Perez JM (2007) The rapid detection and identification of the impurities of simvastatin using high resolution sub 2 micron particle LC coupled to hybrid quadrupole time of flight MS operating with alternating high-low collision energy. J Sep Sci 30(16):2666-2675

28. Hernandez F, Bijlsma L, Sancho JV, Diaz R, Ibanez M (2011) Rapid wide-scope screening of drugs of abuse, prescription drugs with potential for abuse and their metabolites in influent and effluent urban wastewater by ultrahigh pressure liquid chromatography-quadrupole-time-of-flight-mass spectrometry. Anal Chim Acta 684:96-106

29. Bijlsma L, Sancho JV, Hernandez F, Niessen WMA (2011) Fragmentation pathways of drugs of abuse and their metabolites based on QTOF MS/MS and MS(E) accurate-mass spectra. J Mass Spectrom 46:865-875

30. Geromanos SJ, Vissers JPC, Silva JC, Dorschel CA, Li G-Z, Gorenstein MV, Bateman RH, Langridge JI (2009) The detection, correlation, and comparison of peptide precursor and product ions from data independent LC-MS with data dependant LC-MS/MS. Proteomics 9:1683-1695

31. Blech S, Ebner T, Ludwig-Schwellinger E, Stangier J, Roth W (2008) The metabolism and disposition of the oral direct thrombin inhibitor, dabigatran, in humans. J Drug Metab Dispos 36:386-399

32. Blech S, Ludwig-Schwellinger E, Gräfe-Mody EU, Withopf B, Wagner K (2010) The metabolism and disposition of the oral dipeptidyl peptidase-4 inhibitor, linagliptin, in humans. J Drug Metab Dispos 38:667-678

33. Kanu AB, Dwivedi P, Tam M, Matz L, Hill HH (2008) Ion mobility-mass spectrometry. J Mass Spectrom 43(1), and literature cited therein

34. Castro-Perez J, Roddy TP, Nibbering NMM, Shah V, McLaren DG, Previs S, Attygalle AB, Herath $\mathrm{K}$, Chen Z, Wang SP, Mitnaul L, Hubbard BK, Vreeken RJ, Johns DG, Hankemeier $\mathrm{T}$ (2011) Localization of fatty acyl and double bond positions in phosphatidylcholines using a dual stage CID fragmentation coupled with ion mobility mass spectrometry. J Am Mass Spectrom 22:1552-1567

35. Cuyckens F, Wassvik C, Mortishire-Smith RJ, Tresadern G, Campuzano I, Claereboudt J (2011) Product ion mobility as a promising tool for assignment of positional isomers of drug metabolites. Rapid Commun Mass Spectrom 25:3497-3503

36. Hamilton RA, Garnett WR, Kline BJ (1981) Determination of mean serum level by assay of a single pooled sample. Clin Pharmacol Ther 29:250-251

37. Hop CE, Wang Z, Chen Q, Kwei G (1998) Plasma-pooling methods to increase throughput for in vivo pharmacokinetic screening. J Pharm Sci 87:901-903

38. Giles K, Williams JP, Campuzano I (2011) Enhancements in travelling wave ion mobility resolution. Rapid Commun Mass Spectrom 25:1559-1566

39. Pringle SD, Giles K, Wildgoose JL, Williams JP, Slade SE, Thalassinos K, Bateman RH, Bowers MT, Scrivens JH (2007) An investigation of the mobility separation of some peptide and protein ions using a new hybrid quadrupole/travelling wave IMS/ oa-TOF instrument. Int J Mass Spectrom 261:1-12

40. Crecelius AC, Baumgaertel A, Schubert US (2009) Tandem mass spectrometry of synthetic polymers. J Mass Spectrom 44:12771286 